\section{Experiencia participativa orientada a la prevención de la exposición a plaguicidas en la producción hortícola del Cinturón Verde de la ciudad de Córdoba}

\author{
Mariana Andrea Eandi \\ marianaeandi@gmail.com \\ Valeria Cecilia Soria \\ valesoria5@gmail.com \\ Luciana Dezzotti \\ luciana.dezzotti@gmail.com
}

\author{
Mariana Butinof \\ mariana@butinof.com.ar \\ Universidad Nacional de Córdoba, \\ Argentina.
}

Investigación y extensión universitaria / Intervenciones
RECEPCIÓN: 25/09/18

ACEPTACIÓN FINAL: 28/11/18
Participatory experience aimed at the prevention of exposure to pesticides in the horticultural production of the Green Belt of the city of Córdoba

\section{Abstract}

The objective of this communication is to reflect on an experience aimed at promoting risk prevention actions associated with exposure to pesticides in the context of daily life and work of horticulturists and their families in the Green Belt of the City of Córdoba, as well as also to favor dialogues and actions to promote healthy habits and health care in the spaces where they deploy their productive and reproductive activities. It exposes the process of exchange and construction of knowledge, carried out among horticulturists and their families, referring to the sector, teachers, researchers and students of the National University of Córdoba, and other social actors linked to the problem, during 2013-2017. This participatory research - action (IAP) experience was carried out from the collective health proposal. The construction process allowed to resize the complexity of the exposure context from the shared experiences and to build new strategies to prevent health risks.

Keywords: health-disease-care process, exposure to pesticides, Green Belt of Córdoba, participatory experience.
Experiência participativa voltada para a prevenção da exposição a agrotóxicos na produção hortícola do Cinturão Verde da cidade de Córdoba

\section{Resumo}

O objetivo desta comunicação é refletir sobre uma experiência que visa promover ações de prevenção de riscos associados à exposição a agrotóxicos no contexto do cotidiano e do trabalho de famílias e produtores hortícolas do Cinturão Verde da Cidade de Córdoba, bem como promover diálogos e ações de promoção de hábitos saudáveis e atenção à saúde nos espaços onde implantam suas atividades produtivas e reprodutivas. Expõe o processo de troca e construção de conhecimento realizado entre horticultores e seus familiares, professores, pesquisadores e estudantes da Universidade Nacional de Córdoba, e outros atores sociais ligados ao problema, durante o período 2013-2017. Esta pesquisa participativa - experiência de ação foi realizada a partir da proposta de saúde coletiva. O processo de construção nos permitiu redimensionar a complexidade do contexto de exposição a partir das experiências compartilhadas e construir novas estratégias para evitar riscos à saúde.

Palavras-chave: processo saúde-doençacuidado, exposição a pesticidas, Cinturão Verde de Córdoba, experiência participativa. cuidado, exposición a plaguicidas, Cinturón Verde de Córdoba, experiencia participativa.

Para citación de este artículo: Eandi, M.A.; Soria, V.C.; Dezzotti, L. y Butinof, M. (2018). Experiencia participativa orientada a la prevención de la exposición a plaguicidas en la producción hortícola del Cinturón Verde de la ciudad de Córdoba. +E: Revista de Extensión Universitaria, 8(9), juliodiciembre, 183-194. doi: 10.14409/extension.v8i9.Jul-Dic.7855. 


\section{Introducción}

Las transformaciones de la agricultura en los últimos años, tanto intensiva — propia de la horticultura - como extensiva - producción de granos como commodities para exportación-, han sido promotoras de una nueva conflictividad en su articulación con la vida cotidiana de las comunidades (Silvetti y Cáceres, 2015). Estos conflictos se han desarrollado en un marco de débiles políticas de Estado, temor y desconfianza por parte de diferentes actores y sectores de la sociedad preocupados por: a) las potenciales consecuencias sobre la salud y el ambiente derivadas de las prácticas con uso de plaguicidas en las áreas periurbanas, b) el sostenimiento de modos de producción agrícola basados en tecnologías potencialmente nocivas para la salud y el ambiente, y c) la imposibilidad de algunos/as productores/as de realizar transiciones hacia otras formas de producir alimentos.

Con el fin de trabajar en prevención y reducción de la exposición a plaguicidas a nivel individual y familiar en entornos productivos hortícolas y en comunidades colindantes a los cultivos, así como en la elaboración de acciones de vigilancia y cuidado de la salud de las poblaciones antes mencionadas, el Grupo de Epidemiología Ambiental del Cáncer y otras Enfermedades Crónicas (GEACC) ${ }^{1}$ estudia, desde el paradigma de la Salud Colectiva (Paim, 2006; Breilh, 1994), diferentes contextos productivos, entre ellos, el Cinturón Verde de la Ciudad de Córdoba (CVCC). En este marco, se visualizó la necesidad de articular y promover acciones mediante instancias de participación, iniciando en el año 2013 experiencias con horticultores/as, ${ }^{2}$ familias, comunidades próximas, referentes gremiales, efectores de salud de la zona y referentes municipales del Mercado de Abasto de la Ciudad (MAC) principalmente, con la expectativa de facilitar la construcción y apropiación de nuevos conocimientos y modos de trabajar por parte de cada uno de los actores involucrados y, en definitiva, nuevas oportunidades de cuidado de la salud y el ambiente. El presente artículo surge de la necesidad de reflexionar de manera crítica acerca de las experiencias desarrolladas. Así, es objetivo de este texto exponer esa reflexión sobre las sucesivas aproximaciones y propuestas que hemos implementado para la prevención de riesgos asociados a la exposición a plaguicidas en la producción hortícola del CVCC entre los años 2013 y 2017.

En el desarrollo de la experiencia se han mantenido presentes elementos centrales de la Investigación-Acción Participativa (IAP): a) ser una metodología para el cambio, b) fomentar la participación y autodeterminación de los sujetos que la utilizan, y c) ser la expresión de la relación dialéctica entre conocimiento y acción. Elementos que, considerados en su conjunto, permiten configurarla como una herramienta útil de apropiación y de alteración de la realidad para quienes no poseen esa facultad, con un fin centrado en el mejoramiento de sus condiciones de vida (Sirvent y Pigal, 2012; Ortiz y Borjas, 2008; Becerra Hernández y Moya Romero, 2010).

1) Para mayor información: http://inicsa.webs.fcm.unc.edu.ar/lineas-de-investigacion/epidemiologia-ambiental-del-cancer-y-otras-enfermedades-cronicas-en-cordoba/

2) Se denominan en el artículo como horticultores/as a todos/as los trabajadores que participan en la producción de hortalizas en el CVCC, indistintamente de la categoría laboral que posean (propietario, arrendatario, mediero, empleado). 


\section{Producción hortícola en el CVCC como contexto de vida y trabajo para las familias y productores hortícolas}

Argentina, país con una larga tradición agropecuaria, presenta en la producción agrícola una amplia gama de condiciones geográficas y territoriales, las que han propiciado procesos de construcción social diferenciados en el sector. La horticultura se caracteriza por su amplia distribución en el país, una multiplicidad de climas y gran diversidad de los cultivos producidos. En la actualidad abarca alrededor de 700000 hectáreas (Mitideri, 2015) y ocupa cerca de 10000 trabajadores por año, lo que la transforma en una actividad de gran valor social en el país (Bocero y Prado, 2007). La mayor parte de ella se condensa en las cercanías de los grandes centros urbanos: Buenos Aires, Mendoza y Córdoba aportan la mitad del total del volumen producido. Es relevante señalar que el sector no escapa al Modelo Productivo Agrícola Dominante (MPAD), destacándose en el mismo las explotaciones primarias intensivas en cuanto a los factores de producción de la tierra, trabajo, capital y tecnología (Giarracca y Teubal, 2010). Comparado con el resto del sector agropecuario, demanda en su totalidad 30 veces más mano de obra y 20 veces más insumos por unidad de producción, entre ellos, plaguicidas. La presión de los mercados de consumo implica el uso de grandes volúmenes de plaguicidas, lo que implica un riesgo para los consumidores, los trabajadores y el medio ambiente (Babbit, 2010). En este sentido, la Organización Internacional del Trabajo considera la agricultura como uno de los sectores más peligrosos (OIT, 2011), relacionado ello con la diversidad de tareas y situaciones de demanda física a las que se exponen trabajadores/as, a considerar en la prevención de lesiones y accidentes (Mitidieri y Corbino, 2012).

En nuestro país, la horticultura periurbana estuvo marcada desde sus inicios por el carácter familiar de sus explotaciones y la condición de migrantes de los productores. A principios del siglo XX, con la intervención estatal, arribaron familias de nacionalidad italiana, portuguesa y española. A fines del siglo pasado y principios del actual, se produjo una segunda corriente migratoria procedente de Bolivia que conformó las llamadas «migraciones desde abajo", por ser procesos migratorios no programados por el Estado (Smith y Guarnizo, 1997), y que aportaron un cambio en la configuración de las explotaciones hortícolas. Actualmente, el $60 \%$ de los cinturones verdes del país son sostenidos por familias bolivianas, situación que se da también en la ciudad de Córdoba (Benencia y Gazzotti, 1995; Quaranta, 2010). Es importante comentar que ser migrante en Argentina ha sido históricamente un impedimento para la vinculación y acceso al sistema de salud formal ${ }^{3}$ (Ceriani Cernadas y Morales, 2011).

EI CVCC está ubicado en el centro de la provincia, rodeando a la ciudad capital. Cubre actualmente 5500 hectáreas y aporta el 16 \% de la producción hortícola nacional. En la actualidad, se observa una reducción de su superficie y un desplazamiento de la horticultura a otros lugares. Según las estimaciones existentes, ha pasado de tener 11000 hectáreas en 2004 a un área cultivada en el año 2012 de 5500 hectáreas (Secretaría de Comercio Inte-

3) Respecto de los derechos de las personas migrantes en Argentina, Pablo Ceriani Cernadas y Diego Morales (2011) expresan, con relación a la sanción de la Ley 25.871 (2004): "Sin lugar a dudas, el contenido normativo sobre estos derechos [sociales] básicos alcanza un alto nivel de reconocimiento y protección. De todos modos, lo crucial es su efectiva realización, el ejercicio cotidiano de estos derechos por todas y cada una de las personas migrantes que habiten en el país. Para ello, resulta fundamental que las autoridades públicas respeten plenamente estas disposiciones y que se adopten diversas políticas y medidas dirigidas a revertir las prácticas discriminatorias presentes en las últimas décadas". 
rior, 2012), proceso de reducción que se produce en el marco de una intensa puja territorial, donde intervienen medianos y grandes productores de cereales y oleaginosas, el aumento del área urbana, mediada por el mercado inmobiliario, los asentamientos poblacionales informales y el crecimiento del sector industrial, que también avanza sobre el territorio hortícola.

Se conoce que la mayoría de las quintas en el CVCC son explotaciones pequeñas o medianas, de 1 a 20 hectáreas (83,9 \%) (Butinof et al., 2014) y su principal ventaja competitiva es la cercanía al mercado consumidor. La mayoría de las Familias Hortícolas (FH) viven en las quintas $(70 \%)$, principalmente en la zona noreste de la ciudad. Predominan las familias nucleares $(62,5 \%)$, siendo sus miembros activos en la producción y comercialización de alimentos: 30,6 \% de las mujeres trabaja en la quinta y $19 \%$ de sus hijos e hijas también lo hace (Machado et al., 2017). El rol productivo de las mujeres es generalmente invisibilizado, queda encubierto bajo la figura de "ayuda"; sin embargo, algunas mujeres se encuentran a cargo de emprendimientos productivos y asumen todas las tareas del proceso (Dezzotti et al., 2017). Se suman a las labores trabajadores asalariados temporales o permanententes, quienes realizan las tareas más pesadas y son a menudo migrantes indocumentados, ocultos a los ojos del sistema (Machado et al., 2017).

\section{El problema del trabajo con plaguicidas en el entramado entre espacio productivo y reproductivo: ¿es posible pensar la salud en este contexto?}

La mayoría de las $\mathrm{FH}$ viven en el lugar donde se producen alimentos, la casa-hogar-quinta se transforma en un espacio indivisible y sinérgico: el libre tránsito de los integrantes de la familia entre estos ámbitos genera escenarios de exposición continua aun cuando no todos los miembros de la familia tengan contacto directo con los plaguicidas. Las características de la producción determinan que su uso sea continuo, intenso y repetitivo, lo que puede provocar intoxicaciones crónicas o agudas de tipo no intencional o intencional, aunque estas últimas son poco habituales (Fernández et al., 2011). La naturalización de la situación impide tomar medidas para el cuidado de la salud a nivel individual, familiar y colectivo, al no visualizar el trabajo como generador de enfermedad (Machado et al., 2012). La proximidad favorece la exposición debido a la deriva primaria de los plaguicidas (aquella que se produce durante el transporte de los mismos en sitios donde no se aplicó), la deriva secundaria (la volatilización y el movimiento de residuos de plaguicidas desde el suelo, las plantas, el viento y después de la aplicación) y, por último, la ingestión dietética de residuos de los mismos en alimentos o agua "potable" (Deziel et al., 2015). La exposición no ocupacional puede ocurrir mediante una diversidad de mecanismos y vías, afectando al horticultor y demás convivientes. Esto depende, entre otros factores, de las prácticas posaplicación de plaguicidas, entre ellas: la posibilidad de tomar una ducha al finalizar la jornada laboral, cambiarse de ropa en el lugar de trabajo, disponer de espacios y momentos para alimentarse alejados de los cultivos. Se contempla que el ingreso al hogar con la ropa de trabajo, especialmente el calzado, intervenir en la elaboración de alimentos sin aseo, la distancia del hogar a los cultivos asperjados, entre otras situaciones, aumentan el riesgo de exposición para los convivientes (Fenske y Elkner, 1990).

Para los/as trabajadores/as, la magnitud de la exposición a plaguicidas depende de diversos factores, entre los que se incluyen la utilización de Equipo de Protección Personal 
$(E P P)^{4}$ durante las tareas de preparación y aplicación de plaguicidas, al momento de cargar, reparar y limpiar los equipos de aplicación, acción clave en la disminución de la dosis absorbida de los contaminantes y, consecuentemente, de los riesgos y potenciales efectos en salud (Remor et al., 2009; Hines y Deddens, 2001; NIOSH, 1994). Aumentan la posibilidad de exposición: a) la tecnología de aplicación de plaguicidas; en el CVCC es la mochila (77 $\%$ ), que es cargada en la espalda y con altas chances de derrame en el cuerpo; b) el uso de incompleto de EPP por parte de la mayoría de los productores; y c) la utilización promedio de 13 plaguicidas diferentes (Franchini et al., 2016).

Acerca de la percepción de peligrosidad de los plaguicidas, el $70 \%$ de los/as horticultores/as asocia la peligrosidad con los efectos de exposiciones agudas que se expresan con sintomatología irritativa en piel y ojos, principalmente, y no reconoce los riesgos relacionados con la exposición crónica. Asimismo, el nivel de toxicidad de los plaguicidas reconocido por ellos mismos no se encontró vinculado a la adopción de medidas de protección personal (Franchini et al., 2016). Machado et al. (2012) indagaron sobre los mecanismos subjetivos implicados en el cuidado de la salud ante la exposición de los horticultores del CVCC, problematizando las condiciones de vida, percepción de riesgo y su utilidad para analizar las prácticas con plaguicidas. Los resultados muestran que en esta población los problemas de salud no están asociados a la exposición a plaguicidas, obturando la puesta en práctica de mecanismos de autocuidado durante su manipulación. Es relevante exponer que se identifican escasas políticas de salud específicas a esta problemática por parte del sector salud.

El discurso imperante desde el sector productivo propone la implementación de Buenas Prácticas Hortícolas $(\mathrm{BPH})^{5}$ como respuesta eficaz al problema de la exposición, y dicha implementación queda bajo responsabilidad del horticultor, quien no siempre cuenta con la posibilidad de llevarla a cabo. Esta perspectiva ha sido ampliamente criticada por otros autores, que reportaron la insuficiencia de las BPH en la mitigación del riesgo en distintas poblaciones (Weng y Black, 2015). Este abordaje desconsidera la complejidad del problema al no contextualizarlo en los escenarios de la vida cotidiana en que se desarrollan las labores hortícolas. Emergen grandes vacíos que dejan vulnerables a las familias y a las comunidades colindantes (Machado et al., 2017): en la mayoría de los casos, el cuidado de la salud no resulta así jerarquizado ni aun factible.

La utilización de plaguicidas se realiza en presencia de marcos institucionales y jurídicos que establecen garantías a la salud de los habitantes y al ambiente que no siempre son reconocidas en el sector. Además de las Constituciones Nacional ${ }^{6}$ y Provincial y la Ley Nacional de Ambiente, Córdoba cuenta con la Ley Provincial № 9.164, de Productos Químicos

4) El EPP incluye: mameluco impermeable, botas, guantes de nitrilo, sombrero, gafas y máscara con carbón activado. 5) BPH: conjunto de principios, normas y recomendaciones tendientes a reducir los riesgos físicos, químicos y biológicos en la producción, cosecha y acondicionamiento en la producción frutihortícola (Instituto Nacional de Tecnología Industrial, s/f).

6) La Constitución Nacional Argentina, en su artículo $41^{\circ}$, establece: "Todos los habitantes gozan del derecho a un ambiente sano". Ley Nacional General del Ambiente № 25.657 y la Ley Política Ambiental № 10.208 , de la Provincia de Córdoba, reconocen a la salud como un derecho. Esta última se incorpora al marco normativo ambiental preexistente en la provincia, Ley $N^{\circ} 7343$, que define términos centrales a los fines de contribuir a procesos de ordenamiento ambiental del territorio, entre ellos: ambiente urbano y ambiente agropecuario. Asimismo, la Ley Provincial $N^{\circ} 8973$ incorpora en su listado de desechos peligrosos aquellos resultantes de la producción, preparación y utilización de biocidas y productos fitosanitarios, haciendo un aporte a la gestión ambiental de estos. 
o Biológicos de Uso Agropecuario, y su Decreto reglamentario 132/05, sancionados en el año 2004 y 2005, respectivamente. Dicha ley provincial tiene como objeto "proteger la salud humana, los recursos naturales, la producción agropecuaria, la calidad de los alimentos y materias primas frente al impacto que los productos en cuestión pueden causar, contribuyendo además a su trazabilidad y al desarrollo sostenible". Sin embargo, en este ámbito productivo son escasos el conocimiento, la difusión y la discusión de las normativas vigentes y su cumplimiento y existen lagunas legislativas frente a cuestiones particulares del sector hortícola (Ezenga et al., 2015), en tanto que también es insuficiente el control del Estado (Franchini et al., 2016). Este escenario pone en evidencia la transferencia de responsabilidad, un "hacerse cargo" individual y colectivamente de sus situaciones, entre ellas, la salud. En Córdoba existen antecedentes de conflictos con el sector agrícola extensivo que ayudaron a la visibilización de otras problemáticas del sector, así como la presencia ciudadanos autoorganizados en defensa propia frente a la vulneración de sus derechos (Berger y Ortega, 2010).

\section{La necesidad de prevenir y reducir los riesgos asociados a la exposición: ¿cómo, con quiénes, en qué espacios?}

La descripción hasta aquí desarrollada permite reconocer que la salud y las posibilidades de cuidado con que cuentan las FH del CVCC no solo resultan de prácticas individuales llevadas a cabo por horticultores/as, sino que estas prácticas se encuentran insertas en procesos de determinación de la salud dependientes de las características de organización del sector productivo hortícola en el marco del MPAD y las exigencias que este genera, sin control visible por parte del Estado (Eandi et al., 2018). Asimismo, estos procesos complejos de determinación de la salud escapan al alcance general de la norma que rige en la provincia de Córdoba para el control de productos fitosanitarios de uso agrícola (Ley 9.164).

La epidemiología nos puede mostrar quiénes, dónde, cuándo están, o podrían estar, más involucrados en situaciones de riesgo para la salud en el contexto hortícola. Sin embargo, para avanzar en la comprensión de las causas de los problemas descritos y en su transformación, es necesario ir más allá del diagnóstico de situación. Así, se plantearon los siguientes problemas o nudos críticos:

- La naturalización de la condición de producción-génesis de las prácticas laborales riesgosas para la salud de los/as aplicadores/as, de sus familias y comunidades adyacentes a las zonas cultivadas.

- La alta vulnerabilidad que plantea el contexto social-cultural-ambiental y económico en el cual viven muchas de las familias hortícolas impide tomar medidas de cuidado de la salud a nivel individual y colectivo, no visualizándose el mismo como productor de enfermedad.

- Para muchos horticultores y sus familias, la enfermedad es concebida como algo fuera de su control, obturando la puesta en práctica de mecanismos de autocuidado.

- La situación de vida de las familias hortícolas, muchas de ellas migrantes, dificulta su vinculación y acceso al sistema de salud formal.

- La carencia de estrategias de prevención de riesgos específicas para el sector hortícola por parte de los efectores de salud convoca a trabajar de manera participativa reconociendo el marco de interculturalidad en el cual se despliega la problemática. 
- Los conflictos sectoriales relacionados con las actividades agrícolas dificultan el abordaje de la problemática e impiden el desarrollo de acciones preventivas sobre la salud de las personas.

Reconocer las particularidades del territorio ${ }^{7}$ en el que se plantea el problema en estudio y la explicación que cada actor involucrado en la problemática hace de las mismas, posibilita la elaboración de un plan para su abordaje.

La primera aproximación fue en el año 2013 y consistió en el desarrollo de un "dispositivo de prevención" junto a actores ${ }^{8}$ institucionales vinculados al sector hortícola interesados en la promoción de BPH, agrónomos relacionados con el Instituto Nacional de Tecnología Agropecuaria (INTA) y la Agencia para el Desarrollo de la ciudad de Córdoba (ADEC). El contenido de los talleres fue acordado con estos actores y se abordaron la temática de la salud, el trabajo y la prevención. Se completaron seis encuentros con presencia de 20 horticultores/as y miembros de FH en cada uno de ellos (total de participantes: 180). Cada encuentro taller fue realizado en quintas productivas o espacios cercanos a las mismas (como clubes), en lugares y momentos acordados con los horticultores. Esta primera instancia permitió comenzar a establecer vínculos con los horticultores y sus familias en un ambiente informal y descontracturado, así como identificar contenidos útiles para el abordaje de la problemática y desechar otros. La segunda aproximación (2014) fue desarrollada en encuentros en el MAC, por invitación del Ministerio de Agricultura y Ganadería de la provincia de Córdoba, en el marco de los cursos obligatorios para obtener el carné de Aplicador ${ }^{9}$ (Ley 9.164), a fin de dictar los contenidos relacionados con la prevención de la exposición previstos en las normas de buenas prácticas de agricultura. Estos encuentros se caracterizan por ser masivos, lo que posibilitó tomar contacto con un sector importante de la comunidad de horticultores (200 asistentes por encuentro) y su referente gremial. ${ }^{10}$ Estas actividades colectivas fueron acompañadas de la invitación a realizar una evaluación personal de salud en días posteriores al curso. Para concretar lo expuesto, se generaron acuerdos con centros de salud municipales del área del CVCC, con encuentros individuales que incluyeron: consejería en prevención y cuidados de la salud y examen individual clínico y bioquímico para valorar condiciones de salud asociadas a la exposición a plaguicidas (se valoraron 16 horticultores). Esta instancia de trabajo centrada en las condiciones particulares de salud de los/as horticultores/as en encuentros "cara a cara" brindó una nueva perspectiva sobre la problemática en estudio y aportó valiosos elementos de conocimiento acerca del entramado entre la vida cotidiana y las prácticas productivas. La primera aproximación puso en evidencia la necesidad de abordar grupalmente las prácticas productivas del sector para

7) Haesbaert (2004) define territorio como: "Espacio que envuelve una dimensión simbólica, cultural y una dimensión más concreta, de carácter político disciplinar"; y para Souza (2001), el territorio es "un espacio definido y delimitado por y a partir de relaciones de poder". Se consideran ambos conceptos como complementarios.

8) Según Matus, el actor social es "una personalidad, una organización o una agrupación humana que en forma estable o transitoria tiene capacidad de acumular fuerza, desarrollar intereses y necesidades y actuar produciendo hechos en la situación" (1987:744).

9) Los cursos son obligatorios para obtener carnet de aplicador, en los mismos se abordan cuestiones referidas al uso seguro de los productos, entre ellos importancia del uso de EPP y BPH en general.

10) Asociación de Productores Hortícolas de la Provincia de Córdoba (APRODUCO). 
promover su resignificación entre los/as horticultores/as. La segunda, la necesidad de abordar tales prácticas vinculadas a aquellas de la vida cotidiana.

Durante los años 2014 y 2015 se realizaron visitas a quintas y encuentros con diferentes miembros de la FH del CVCC, de las cuales participaron 14, con el objetivo de observar en contexto el trabajo hortícola, lugar donde se despliegan las prácticas productivas y reproductivas diarias. Estas instancias fueron significativas por el encuentro y reconocimiento del "otro", la generación de lazos de confianza y empatía, elementos centrales para comprender sus tiempos, espacios, lazos establecidos al interior de la familia y entre miembros de la comunidad y aquellos factores que les impiden cuidar su salud en el marco de la producción de alimentos, entendiendo que todas sus actividades diarias giran en torno a la quinta, el trabajo, la producción y la venta de lo producido (Dezzotti y col., 2017).

En 2016 se estableció un acuerdo de colaboración entre el equipo de investigación y APRODUCO orientado al cuidado de la salud de los horticultores y la implementación de $\mathrm{BPH}$ para la mejora de la calidad de los alimentos producidos en materia de seguridad alimentaria $^{11}$ que se desarrolló en el MAC. Este espacio de comercialización cuenta con 8 naves (grandes galpones) con divisorios distintivos según tipo y procedencia de los productos. En este espacio, los horticultores pasan largas horas del día descargando, ordenando y luego vendiendo los alimentos. Se pudo identificar mediante observaciones sucesivas que existen momentos destinados al esparcimiento antes de la venta entre cada una de las actividades anteriores, y este tiempo de "recreación" es valorado como "muy importante" para la socialización, la charla y la escucha. El MAC cuenta con un Centro de Salud perteneciente al municipio de Córdoba ubicado en el lugar entre las naves de venta, donde trabajan un médico y un enfermero que fueron invitados a participar de la propuesta; también en este ámbito existe un aula de uso colectivo donde se realizaron encuentros-taller del colectivo de horticultores del MAC, efectores de salud, referentes gremiales e ingenieros vinculados con el municipio. Se planificaron seis talleres a llevarse a cabo en el aula disponible en el MAC en días y horarios acordados con APRODUCO, de los cuales se implementaron solo los tres primeros debido a la baja concurrencia a los mismos (entre cuatro y seis participantes por taller). Ello motivó un nuevo proceso de indagación acerca de la metodología de trabajo, en el transcurso del cual se dedicó mucho tiempo a recorrer las naves y establecer nuevos encuentros y conversaciones informales con los horticultores. Como resultado de las instancias previas, se construyó una bitácora de reconocimiento de actividades realizadas en la quinta que permite objetivar momentos de trabajo y exposición a plaguicidas, denominada "Diario de Registro de Prácticas". El proceso, apropiación y puesta en práctica del dispositivo de prevención emergente se acompañó en dos lugares: casa-hogar-quinta y el MAC. Esta modalidad nos permitió compartir y generar/fortalecer lazos con horticultores y FH en sus espacios de vida cotidiana, siendo la casa-hogar-quinta el lugar preferido para abordar las problemáticas referidas a la producción hortícola y la vida.

11) Pio — Proyectos de Investigación Orientados— del Ministerio de Ciencia y Tecnología de la Provincia de Córdoba: Exposición ocupacional a plaguicidas entre trabajadores y trabajadoras del Cinturón Verde de Córdoba. Evaluación de estrategias de prevención, buenas prácticas hortícolas y monitoreo de la exposición. 


\section{Conclusiones y desafíos vigentes}

Las sucesivas aproximaciones enunciadas a lo largo de este trabajo posibilitaron reconstruir el escenario de exposición a plaguicidas en el CVCC en el marco de los procesos productivos y reproductivos de las $\mathrm{FH}$, reconociendo al contexto hortícola del CVCC como multidimensional y complejo. Las múltiples aproximaciones permitieron la puesta en marcha de diferentes estrategias para propiciar el encuentro y participación a fin de reducir riesgos y promover transformaciones.

Analizar los modos de producción del conocimiento también requiere observar los procesos metodológicos para su construcción. Los encuentros y diálogos entre actores sociales participantes en la vida cotidiana de las comunidades: horticultores, $\mathrm{FH}$, referentes gremiales, efectores de salud, INTA, ADEC, el Estado y la dialéctica existente entre ellos -y nosotros-, con las actividades de producción en la quinta como mediadoras, hiceron factible reconocer saberes y construir colectivamente conocimientos útiles a los fines de prevenir daños a la salud. Las propuestas alternativas que se fueron implementando surgieron de la necesidad de comprender a la salud en forma histórica y contextualizada, de modo de posibilitar cambios en el escenario de trabajo. Vivenciar el concepto de proceso como un camino cambiante, con momentos de incertidumbre, e ir avanzando en un constante aprendizaje de aciertos y desaciertos, ha conseguido que se siga en el camino iniciado, que se ponga en juego el ejercicio de la flexibilidad, adaptabilidad y evaluación —reflexión constante sobre las diferentes dificultades propias del escenario social y cultural en el que se intervino-. El trabajo colectivo enriqueció la tarea, facilitó el proceso de comprensión de la complejidad del problema y redefinió las preguntas a abordar y las acciones a desarrollar. Desde la consideración de distintos agentes y universos de sentido construidos en torno a las tecnologías de trabajo y el uso de los plaguicidas, acercó al equipo a nuevas interpretaciones en el marco del complejo entramado histórico, social, cultural, económico y político, que rodea esta problemática.

El abordaje de la problemática expuesta no puede quedar escindido del escenario en el cual se desarrolla, lo cual plantea repensar las acciones, resignificar el dónde, el cómo y el para qué. Así, nuestro desafío ha sido el de romper y superar los modelos de abordaje que se han venido desarrollando sobre los procesos de salud/enfermedad/atención, tanto de las ciencias médicas ${ }^{12}$ como de las ciencias sociales. ${ }^{13}$ Todo esto asumiendo que históricamente las relaciones culturales han funcionado en contextos de dominación, de explotación, de hegemonía/subalternidad, en procesos en los cuales operan en forma conjunta sujetos diferenciados cultural y socialmente. Para ello, se propiciaron instancias de diálogo con las familias y comunidades, se establecieron mecanismos de comunicación que permitieran po-

12) Con una impronta biomédica, donde el componente cultural y el social son minimizados o marginados de dichos procesos; corrientes modernas tratan de imbricar algunos contenidos en la "competencia cultural", en términos de asegurar el conocimiento de las taxonomías o las características los grupos y minorías, quedando generalmente los sujetos reducidas y determinados por las características; lo que no modifica sustancialmente el modelo, más bien lo reproduce.

13) Desde el sector de las ciencias sociales, más específicamente la antropología, la interculturalidad se reduce a la relación grupos amerindios-servicios biomédicos, dejando de lado, o dándole muy escaso espacio en sus etnografías, a las diferentes formas de atención a los padecimientos que están operando en los contextos donde vive la población estudiada. 
ner el problema en palabras priorizando las vivencias cotidianas de $\mathrm{FH}$ y horticultores referidas al tema en cuestión. Estos espacios fueron concebidos como el lugar de articulación del saber técnico (BPH, uso de EPP, entre otros) con el saber popular (ideas y significados acerca de los plaguicidas y del cuidado de la salud) para la construcción de aprendizajes mutuos, reconociendo las mutuas competencias y saberes, capacidades y limitaciones, orientados a interrogar la realidad e interpelar los conocimientos previos de unos y otros desde el encuentro, para crear en conjunto estrategias orientadas al accionar.

Acerca del camino recorrido, rescatamos la necesidad de una concepción integral de los procesos de trabajo con un otro, individual y colectivo, y la intencionalidad transformadora de las intervenciones. Los actores sociales son concebidos como sujetos protagonistas de dichas transformaciones en donde su historia, contexto social y cultural son centrales. Por ello el eje estructurante de los trabajos debe ser la vinculación de la teoría con la práctica y la transformación como telón de fondo. Así, detenerse a reflexionar sobre los procesos de trabajo y su devenir, en un marco compartido, resulta fundamental. Sistematizar las experiencias significa entender e interpretar lo que está aconteciendo a partir de un ordenamiento y reconstrucción de los distintos elementos objetivos y subjetivos del proceso para comprenderlo, interpretarlo y aprender de nuestra propia práctica.

El desafío es generar acuerdos intersectoriales que aporten a la modificación de las prácticas productivas vigentes a partir de la transformación del modelo productivo que las determina y obtura, gestar transiciones hacia otras formas de producción menos dañinas para la salud y el ambiente.

\section{Referencias bibliográficas}

Babbit, S. (2010). El periurbano Sampedrino: un espacio de viveros. En Neiman, G. (Ed.). Globalización y Agricultura periurbana en Argentina (pp. 121-135). Facultad Latinoamericana de Ciencias Sociales (FLACSO). Recuperado de http://www.flacso.org.ar/wp-content/uploads/2013/11/Globalizacion-y-agricultura-periurbana-en-la-Argentina.pdf (consultado el 12/06/2018).

Becerra Hernández, R. y Moya Romero, A. (2010). Investigación-acción participativa, crítica y transformadora. Un proceso permanente de construcción. Integra Educativa, (2), 133-156.

Benencia, R. y Gazzotti, A. (1995). Migración limítrofe y empleo: precisiones e interrogantes. Estudios Migratorios Latinoamericanos, 10(31), 513-609,

Berger, M. y Ortega, F. (2010). Poblaciones expuestas a agrotóxicos: autoorganización ciudadana en la defensa de la vida y la salud, Ciudad de Córdoba, Argentina. Physis: Revista de Saúde Coletiva, 20(1), 119-143. Recuperado de https://dx.doi.org/10.1590/S0103-73312010000100008

Bocero, S. y Prado, P. (2007). Horticultura y territorio. Configuraciones territoriales en el cinturón hortícola marplatense a fines de la década del noventa. Estudios Socioterritoriales, (7), 98-119. Recuperado de http:// pdfhumanidades.com/sites/default/files/apuntes/90\%20-\%20Art\%C3\%ADculo\%20BOCERO.DOC

Breilh J. (1994). Dialéctica de lo colectivo en epidemiologia. En Costa, M.F.L. \& Souza, R.P. (Orgs.). Qualidade de vida: compromisso histórico da epidemiologia (pp. 135-145). Belo Horizonte: Coopmed.

Butinof, M.; Fernández, R.; Lantieri, M.J.; Stimolo, M.I.; Blanco, M.; Machado, AL.; (...) Díaz, M.P. (2014). Pesticides and Agricultural Work Environments in Argentina. En Larramendy, M.L. \& Soloneski, S. Pesticides - Toxic Aspects (pp. 115-138).

Ceriani Cernadas, P. y Morales, D. (2011). Argentina: avances y asignaturas pendientes en la consolidación de 
una política migratoria basada en los derechos humanos. Centro de Estudios Legales y Sociales (CELS). Recuperado de https://www.cels.org.ar/web/wp-content/uploads/2011/02/Argentina.-Avances-y-asignaturas-pendientes-en-la-consolidacion.pdf (consultado el 12/05/2017).

Dezzotti, L.; Eandi, M.; y Butinof, M. (2017). Exposición a plaguicidas: prácticas productivas, reproductivas y vida cotidiana de las familias hortícolas. Córdoba, Argentina. X Jornadas Internacionales de Salud Pública. Comunicaciones Orales. Revista de Salud Pública, Ed. Especial, 2106, 22. Recuperado de https://revistas.unc. edu.ar/index.php/RSD/article/viewFile/17095/16716 (consultado el 07/07/2017).

Deziel, N.; Friesen, M.; Hoppin, J.; Hines, C.; Thomas, K.; y Beane Freeman, L. (2015). A Review of Nonoccupational Pathways for Pesticide Exposure in Women Living in Agricultural Areas. Environmental Health Perspectives, 123(6), 515-524. Recuperado de http://ehp.niehs.nih.gov/wp-content/uploads/123/6/ehp.1408273. alt.pdf (consultado el 05/03/2017).

Eandi, M.A.; Franchini, G.; Fernández, R.; Blanco, M.; Dezzotti, L. y Butinof, M. (marzo de 2018). Riesgo de accidente con plaguicidas en las prácticas hortícolas del cinturón verde de Córdoba, Argentina. Anais do IX Congresso Brasileiro de Epidemiologia. Brasil.

Ezenga, M.; Viotti, N.; Eandi M.A y Butinof, M. (2015). Políticas de salud y protección de la salud de poblaciones expuestas a plaguicidas en la Provincia de Córdoba. Tesis de grado. Escuela de Nutrición, FCM, UNC, Córdoba, Argentina.

Fenske, R.A. y Elkner, K.P. (1990). Multi-route exposure assessment and biological monitoring of urban pesticide applicators during structural control treatments with chlorpyrifos. Toxicol Ind Health, (6), 349-371.

Fernández, R.A.; Butinof, M.; Lantieri, M.J.; Stimolo, M.I.; Blanco, M.; Machado, A.L. y Díaz, M.P. (2011). Condiciones de Salud de los Aplicadores de Plaguicidas de la Provincia de Córdoba, Argentina. Un Aspecto del Impacto de la Agriculturización en la Provincia. En XI Jornadas Argentinas de Estudios de Población. Neuquén, Argentina.

Franchini, G.; Butinof, M.; Blanco, M.P.; Machado, A.L.; Fernández, R.A. y Díaz, M.P. (2016). Occupational risks associated with the use of pesticides in the green belt of Córdoba, Argentina. Acta toxicológica argentina, 24(1), 5867. Recuperado de http://www.scielo.org.ar/scielo.php?script=sci_arttext\&pid=S1851-37432016000100007\&Ing=es\&tlng=en (consultado el 29/06/2018).

Giarracca, N. y Teubal, M. (2010). Disputas por los territorios y recursos naturales: el modelo Extractivo. ALASRU Nueva Época, 5, 113-130. Recuperado de https://www.academia.edu/24856532/Disputas_por_los_territorios_y_recursos_naturales_el_modelo_extractivo (consultado de 15/05/2018).

Haesbaert, R. (2004). O mito da desterritorializaçao: do fim dos territórios á multiterritiralidade. Rio de Janeiro: Bertrand.

Hines, C. y Deddens, J. (2001). Determinants of chlorpyrifos exposures and urinary 3,5, 6-trichloro-2-pyridinol levels among termiticide applicators. Oxford University Press. Annals Occupationals Hygiene, 45(4), 309-321. Instituto Nacional de Tecnología Industrial. Organismo de Certificación (s/f). Certificación campo voluntario de procesos. Certificación de Buenas Prácticas Agrícolas. Recuperado de https://www.inti.gob.ar/certificaciones/cBPAgricolas.htm (consultado el 06/11/2016).

Machado, A.L.; Ruiz, M.V.; Sastre, M.A.; Butinof, M.; Blanco, M.; Lantieri, M.J.; (...) Díaz M.P. (2012). Exposición a plaguicidas, cuidado de la salud y subjetividad. Revista Kairos, 16(30), 1-17. Recuperado de http:// www.revistakairos.org/wp-content/uploads/Machado.pdf (consultado el 24 /03/2018).

Machado, A.L.; Butinof, M.; Eandi, M.A.; Portilla, A.M.; Fernández, R.A.; Soria, V. y Franchini, G. (2017). Vulnerabilidad y riesgo por plaguicidas en horticultura del cinturón verde en Córdoba, Argentina. Rev. Fac. Nac. Salud Pública, 35(1), 97-108. doi:10.17533/udea.rfnsp.v35n1a11

Matus, C. (1987). Política, Planificación y Gobierno. Caracas: ALTADIR. 
Mitidieri, M. y Corbino, G. (2012). Manual de horticultura periurbana. National Institute of Agricultural Technology. Recuperado de http://inta.gob.ar/documentos/manual-de-horticultura-periurbana1/at_multi_download/ file/Manual\%20de\%20horticultura\%20urbana\%20y\%20periurbana.pdf (consultado el 09/04/2015).

Mitidieri, M. (2015). Programa Nacional Hortalizas, Flores y Aromáticas Plan de Gestión Integrador (PNHFA). Contribución al desarrollo territorial de las producciones intensivas. INTA San Pedro. Recuperado de https:// inta.gob.ar/sites/default/files/script-tmp-plan_de_gestin_pnhfa_1106081.pdf (10/04/2017)

NIOSH (1994). Application Manual for the Revised NIOSH Lifting Equation. CDC: National Institute for Occupational and Safety Health, 94-110.

OIT (2011). Informe sobre el Trabajo en el Mundo 2011: Los mercados al servicio del empleo. Geneva: ILO. Recuperado de http://www.ilo.org/global/publications/ilo-bookstore/order-online/books/WCMS_166021/lang--es/ index.htm (consultado el 10/05/2017).

Ortiz, M. y Borjas, B. (2008). La Investigación Acción Participativa: aporte de Fals Borda a la educación popular. Espacio Abierto, 17(4), 615-627. Recuperado de http://www.redalyc.org/articulo.oa?id=12217404 (consultado el 25/11/2017).

Paim, J.S.; Teixeira, C.F. (2006). Política, planejamento e gestão em saúde: balanço do estado da arte. Rev. Saúde Pública, (40), 73-78. São Paulo.

Quaranta, G. (2010). Estructura ocupacional, características de la demanda y perfil de la oferta laboral en el agro argentino a principios de la década actual. En Neiman, G. (Ed.). Estudios sobre la demanda de trabajo en el agro argentino. Buenos Aires: Ciccus.

Remor, A.P.; Caprini Totti, C.; Alves Moreira, D.; Pimentel Dutra, G.; Dahlström Heuser, V. y Marlei Boeira, J. (2009). Occupational exposure of farm workers to pesticides: Biochemical parameters and evaluation of genotoxicity. Environ. Internat, 35, 273-278.

Secretaria de Comercio Interior (2012). Corporación del mercado central de Buenos Aires. La producción de hortalizas en Argentina. Recuperado de http://www.mercadocentral.gob.ar/ziptecnicas/la_produccion_de_ hortalizas_en_argentina.pdf (consultado el 21/07/2013).

Silvetti, F.; Cáceres, D. (2015). La expansión de monocultivos de exportación en Argentina y Costa Rica. Conflictos socioambientales y lucha campesina por la justicia ambiental. Mundo Agrario, 16(32). Recuperado de http://www.mundoagrario.unlp.edu.ar/article/view/MAv16n32a08 (consultado el 22/08/2017).

Sirvent, M.T.; y Pigal, L. (2012). Investigación acción participativa. Proyecto Páramo Andino. Recuperado de www.flacsoandes.edu.ec (consultado el 25/11/2017).

Smith, M.P. y Guarnizo, L.E. (1998). In Transnationalism from Below, Comparative Urban and Community Research, New Brunswick. NJ: Transaction Publishers, (6), 1-33.

Souza, M.J.L. (2001). O território: sobre espaço e poder. Autonomia e Desenvolvimento. Em Castro, I.E.; Da C. Gomes, P.C. y Correa, R.L. (Eds.). Geografia: conceitos e temas. Rio de Janeiro: Bertrand.

Weng C.Y.; Black C. (2015) Taiwanese farm workers' pesticide knowledge, attitudes, behavior sand clothing practices. Int J EnvironHeal R., 25(6), 685-696. doi: http://doi.org/10.1080/ 09603123.2015.1020415.

\section{Leyes}

Secretaría de Agricultura y Ganadería. Legislatura de la Provincia de Córdoba, Argentina. Ley 9.164. Ley de Productos Químicos y Biológicos de Uso Agropecuario, 2 de junio de 2004. 